\title{
Lamellar thickness in semicrystalline polymers as a result of the competition between crystal growth and intracrystalline chain dynamics
}

\section{Martha Schulz}

Martin-Luther Universität Halle-Wittenberg https://orcid.org/0000-0002-0506-165X

\section{Mareen Schäfer}

Martin-Luther Universität Halle-Wittenberg

\section{Kay Saalwächter}

Martin-Luther-Universität Halle-Wittenberg https://orcid.org/0000-0002-6246-4770

Thomas Thurn-Albrecht ( $\nabla$ thurn-albrecht@physik.uni-halle.de)

Martin-Luther Universität Halle-Wittenberg https://orcid.org/0000-0002-7618-0218

\section{Article}

Keywords: lamellar crystals, semicrystalline polymers, poly(oxymethylene)

Posted Date: July 9th, 2021

DOI: https://doi.org/10.21203/rs.3.rs-638783/v1

License: (c) (i) This work is licensed under a Creative Commons Attribution 4.0 International License.

Read Full License

Version of Record: A version of this preprint was published at Nature Communications on January 10th, 2022. See the published version at https://doi.org/10.1038/s41467-021-27752-0. 


\title{
Lamellar thickness in semicrystalline polymers as
}

\section{a result of the competition between crystal} growth and intracrystalline chain dynamics

\author{
Martha Schulz, Mareen Schäfer, Kay Saalwächter, ${ }^{*}$ and Thomas \\ Institut für Physik, Martin-Luther-Universität Halle-Wittenberg, von-Danckelmann-Platz 3,

\begin{abstract}
Thurn-Albrecht*
06120 Halle, Germany
\end{abstract}

E-mail: kay.saalwaechter@physik.uni-halle.de; thomas.thurn-albrecht@physik.uni-halle.de

\begin{abstract}
The non-equilibrium thickness of lamellar crystals in semicrystalline polymers varies largely between different polymer systems and depends on the crystallization temperature $T_{c}$. There is currently no consensus on the mechanism of thickness selection. Previous work has highlighted the decisive role of intracrystalline chain dynamics (ICD) in special cases, but a systematic dependence of lamellar thickness on relevant timescales such as that of ICD and stem attachment has not yet been established. Studying the morphology by small-angle X-ray scattering and the two timescales by NMR methods and polarization microscopy, we here present data on poly(oxymethylene), a case with comparably slow ICD. It fills the gap between previously studied cases of absent and fast ICD, enabling us to establish for the first time a quantitative dependence of lamellar thickness on the competition between the noted timescales.
\end{abstract}




\section{Introduction}

The characteristic morphological feature of semicrystalline polymers crystallized from the melt is a nanoscopic two-phase structure of thin lamellar crystals separated by disordered amorphous layers, which contain the entanglements retained during crystallization. This morphology is to a large extent responsible for the advantageous mechanical properties of semicrystalline polymers ${ }^{1}$. It has been a classical question in polymer physics, which factors control the thickness of the crystalline layers resulting in a number of crystallization models without reaching final consensus ${ }^{2,3}$.

Most crystallization models start from the assumption that the semicrystalline morphology is a non-equilibrium structure, which is experimentally supported by the observation of a melting point depression that depends on thermal history, specifically the crystallization conditions. Structurally the melting point depression is explained by a finite crystal thickness ${ }^{1}$. In consequence, for a given crystallization temperature $T_{c}$ there is a minimal stable crystal thickness. To explain the selection of a relatively well-defined crystal thickness during crystallization, a second criterion defining an upper limit for the thickness is required. At this point the assumptions made by different models diverge. The classical approach assumes that the crystal thickness is kinetically selected. The crystals with the thickness that grow the fastest, dominate ${ }^{4-8}$, and once a stable crystal has formed, it is assumed that no further structural changes will take place. Multistage models on the other hand assume that crystal growth happens in several stages and is coupled to crystal reorganization processes. Different mechanisms have been suggested -without reaching final agreement- to limit reorganization to a certain thickness, as thickness dependent stability of different crystal phases ${ }^{9}$ or mesophases ${ }^{10,11}$ or thickness dependent intracrystalline chain dynamics ${ }^{3,12,13}$. All these models primarily aimed at an explanation of the temperature dependence of the crystal thickness of a given semicrystalline polymer. They disregard to the most part variations of crystal thickness between different polymers as well as the question what determines the thickness of the amorphous layers and therefore the overall crystallinity. 
In view of this incomplete understanding, we started a series of investigations with the aim of providing a broader perspective on the formation of the semicrystalline morphology by comparing polymers with different intracrystalline chain dynamics. Our starting point was an old observation by Boyd ${ }^{14,15}$ that relates the crystallinity of polymers to the existence of a so-called $\alpha_{c}$-relaxation process. These relaxation processes are a unique feature of polymer crystals and originate from conformational defects moving through the crystals. They enable intracrystalline chain dynamics (ICD), as shown later directly by advanced NMR-methods ${ }^{16}$. Generally, polymers with ICD (crystal-mobile) show a higher crystallinity (> 50\%) than polymers without ICD (crystal-fixed). For a specific case, an important contribution of the ICD to the crystal thickness for the case of poly(1-butene) was suggested. This polymer shows two crystal structures, of which one is crystal-fixed the other one crystal-mobile with a fast ICD. ${ }^{17,18}$ The relevant observation was that direct crystallization into the crystal-fixed form I, either by crystallization from solution or by choosing a sample with tacticity defects, led to much thinner crystals than the usual pathway, in which crystallization proceeds via the crystal-mobile form II, followed by a solid-solid transition into form $I^{18,19}$. For the latter case, the crystal thickness also showed a stronger dependence on the crystallization temperature, presumably caused by the stronger effect of ICD at high temperatures. However, the question what finally limits the crystal thickness was not specifically addressed.

Previously, we systematically compared a pair of crystal-fixed and crystal-mobile model polymers making use of new experimental developments in SAXS, NMR and DSC. Our experiments led us to the hypothesis that generally, the morphology of semicrystalline polymers results from the interplay or competition between kinetics of crystal growth and ICD leading to different morphological characteristics of crystal-fixed and crystal-mobile polymers ${ }^{20}$. The crystallization of a crystal-fixed polymer like PCL results in the formation of marginally stable crystallites of well-defined thickness, which reorganize constantly during heating. We could later on confirm this result by fast scannning calorimetry ${ }^{21}$. A crystalmobile polymer like PEO on the other hand shows a well-defined thickness of the amorphous 
regions and crystalline lamellae that are stable over a large temperature range. Detailed analysis of NMR data reflecting the time-scale of ICD in the temperature range of crystallization showed that indeed, for PEO the ICD is so fast that it can cause reorganization over a very small nanometre-sized reorganization zone directly behind the growth front and practically simultaneously with crystal growth ${ }^{20}$. From these results we concluded that in crystal-mobile polymers the morphology is controlled by a minimum value of the amorphous thickness related to the entanglement density in the amorphous regions.

In order to enable a more quantitative description of the above-mentioned competition between crystal growth and ICD we introduced three parameters describing the typical timescales. As depicted in Figure 1(A) below, we describe the timescale of crystallization by the layer crystallization time $\tau_{l c}$, the time during which the crystal grows on average by one molecular layer. $\left\langle\tau_{c}\right\rangle$ and $\tau_{\text {stem }}$ on the other hand are the characteristic timescales of the ICD. Here $\left\langle\tau_{c}\right\rangle$ is the so-called jump correlation time as probed by NMR, i.e. the average time between two helical defect jumps, whereas $\tau_{\text {stem }}$ represents the time, during which a defect diffuses over a distance equal to the crystal thickness $d_{c}$ by successive helical jumps. Our previous experiments on PCL and PEO correspond to the cases of non-existing (or very slow) and very fast ICD, i.e. $\left\langle\tau_{c}\right\rangle \gg \tau_{l c}$ and $\left\langle\tau_{c}\right\rangle \ll \tau_{l c}$ respectively. $\tau_{c}$ is measured on the fully crystallized sample. As we cannot exclude that the ICD is faster directly behind the growth front, the measured $\tau_{c}$ is an upper estimate for the relevant parameter, but this does not harm the arguments in general.

Here, we present a set of experiments designed as a critical test of the hypothesized competition between crystal growth and ICD by extending previous our studies to a polymer with ICD on a intermediate timescale, namely poly(oxymethylene) (POM). This choice of sample enables us to establish a quantitative dependence of lamellar thickness on the competition between the noted timescales. In such a case we expect intermediate crystal thicknesses, additionally the opposite temperature dependence of $\left\langle\tau_{c}\right\rangle(T)$ and $\tau_{l c}(T)$ should play an important role. Furthermore, we extend our previous static SAXS experiments to 
time-dependent measurements using a position dependent detector, which enables us to observe thickening of lamellar crystals directly during different stages of crystallization. The timescales of crystal growth and ICD are characterized by optical microscopy and solid state NMR, respectively.

\section{Results}

\section{Characteristic timescales}

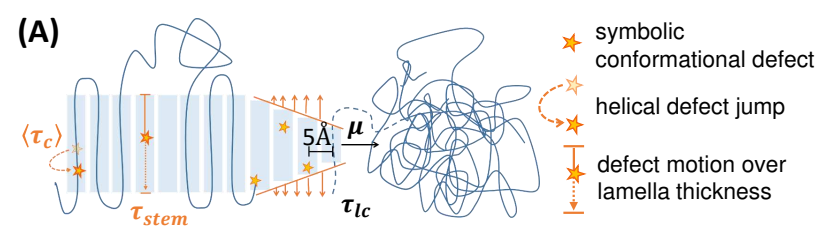

(B)

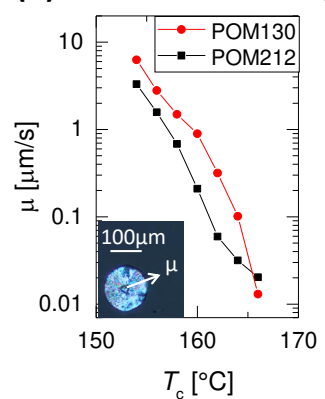

(C)

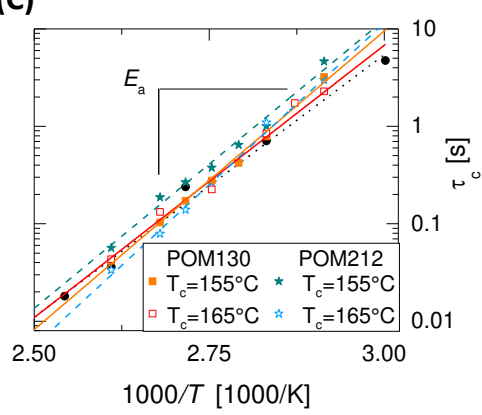

Figure 1: Competing timescales of crystal growth $\left(\tau_{l c}\right)$ and intracrystalline chain dynamics $\left(\left\langle\tau_{c}\right\rangle, \tau_{\text {stem }}\right)$ (A) Schematic illustration of the crystallization process with $\tau_{l c},\left\langle\tau_{c}\right\rangle$, and $\tau_{\text {stem }}$. (B) $\mu$ of poly(oxymethylene) (POM) as function of crystallization temperature $T_{c}$. The inset illustrates how $\mu$ is determined from the growth of spherulites during isothermal crystallization. (C) Arrhenius temperature dependence of the jump correlation time $\left\langle\tau_{c}\right\rangle$ for two samples of POM isothermally crystallized at different $T_{c}$.

We start with the determination of the characteristic times in POM. Following ref. ${ }^{20}$ the layer crystallization time $\tau_{l c}$, during which a crystal grows on average over a distance corresponding to one molecular layer, can be calculated from the crystal growth velocity $\mu$,

$$
\tau_{l c}=\frac{5 \AA}{\mu}
$$


assuming a typical intermolecular distance of about $5 \AA$. $\mu$ was measured by optical microscopy. Figure 1 (B) shows $\mu$ as a function of $T_{c}$ for POM130 and POM212 (cf. Table 1). Corresponding data for PCL and PEO were already published and can be found in the SI.

Previous investigations suggested that POM belongs to the class of crystal-mobile polymers, and that its intracrystalline chain dynamics (ICD) is much slower than in $\mathrm{PEO}^{16}$. Most of the corresponding experiments were performed in the 1960s by mechanical and dielectric measurements, resulting in a wide range of reported activation energies from $E_{a}=88 \mathrm{~kJ} / \mathrm{mol}$ to $328 \mathrm{~kJ} / \mathrm{mol}^{22-27}$. Schmidt-Rohr and Spiess reported the first NMR-based value of $E_{a}=(83 \pm 8) \mathrm{kJ} / \mathrm{mol}$ for a not further specified POM-homopolymer ${ }^{28}$. Additional investigations on the copolymer Hostaform were performed by Kentgens using 2D Exchange NMR measurements and Karahaliou using dielectric measurements. They reported values of $E_{a}=(83 \pm 68) \mathrm{kJ} / \mathrm{mol}^{29}$ and $E_{a}=83 \mathrm{~kJ} / \mathrm{mol}$ to $100 \mathrm{~kJ} / \mathrm{mol}^{30}$.

To provide a detailed and reliable characterization of the ICD for the same samples as used for the structural analyis, we performed NMR experiments on isothermally crystallized POM samples, using the ${ }^{13} \mathrm{C}$ MAS CODEX technique ${ }^{31}$, which probes slow segmental reorientations. The analysis of NMR spectra measured at different temperatures (here $70^{\circ} \mathrm{C}$ - $110^{\circ} \mathrm{C}$ ) allows the determination of $\left\langle\tau_{c}\right\rangle(T)$ and of the activation energy describing its temperature dependence.

$$
\left\langle\tau_{c}\right\rangle=\tau_{0} \cdot \exp \frac{E_{a}}{R T}
$$

$E_{a}$ and $\tau_{0}$ were determined for different $T_{c} \mathrm{~s}$ and both molecular weights. Exemplary results are shown in Figure 1(C), the full set of resulting values are listed in Table S1 in the SI. As the samples with different $T_{c}$ have different crystal thickness $d_{c}$ (see below) this analysis also reveals if $\left\langle\tau_{c}\right\rangle$ depends on $d_{c}$. The activation energies vary by about $10 \%$ from sample to sample, however we could not observe a systematic dependence of $\left\langle\tau_{c}\right\rangle$ on $d_{c}$, different from the case of $\mathrm{PEO}^{32}$. Our data do not allow for conclusions on a potential molecular weight 
effect. The average values are $E_{a}=113 \mathrm{~kJ} / \mathrm{mol}$ and $\tau_{0}=1.4 \times 10^{-16} \mathrm{~s}$ for POM130 and $E_{a}=117 \mathrm{~kJ} / \mathrm{mol}$ and $\tau_{0}=1.6 \times 10^{-15} \mathrm{~s}$ for POM212. For consistency we cross-checked the results by dynamic mechanical measurements and found similar results. Details are given in the SI.

The typical time range of crystal reorganization can be estimated from the correlation time $\tau_{c}$ measured by NMR. $\left\langle\tau_{c}\right\rangle$ corresponds to the average residence time of the chain in a given helical raster. ${ }^{32}$ For an $n_{m}$-helix with $n$ monomers per $m$ turns over a lattice distance c, the corresponding jump distance is $\Delta z_{c}=c / n\left(\Delta z_{c}=0.279 \mathrm{~nm}\right.$ for the $7_{2}$ helix in $\mathrm{PEO}^{33}$ and $\Delta z_{c}=0.192 \mathrm{~nm}$ for the $9_{5}$ helix in $\left.\mathrm{POM}^{34}\right) .{ }^{1}$ We estimate the time $\tau_{\text {stem }}$, within which a chain in the crystal diffuses over a distance equal to the crystal thickness by successive helix jumps as

$$
\tau_{\text {stem }} \approx\left\langle\tau_{c}\right\rangle \cdot d_{c}^{2} / \Delta z_{c}^{2}
$$

Here we assumed a random walk of $N=\tau_{\text {stem }} /\left\langle\tau_{c}\right\rangle$ steps of size $\Delta z_{c}$. The squared distance travelled is $d_{c}^{2}$. For $d_{c}$ we use the values of the lamellar thickness obtained by SAXS measurements after isothermal crystallization as shown below. The corresponding data are listed in the SI. Eq. (3) is an approximation for the early stage of growth, during which constraints by neighboring lamellar crystals are still weak.

Together with previously published data for PEO with fast ICD and PCL, for which we could exclude any dynamics up to a timescale of $1 \mathrm{~s}$, we can now compare the timescales for ICD (range between $\tau_{c}$ and $\tau_{\text {stem }}$ ) and crystal growth $\left(\tau_{l c}\right)$ in the temperature range of crystallization for all three polymers. ${ }^{20,32,35}$ The result is shown in Figure 2. For PCL we used the NMR detection limit for ICD as a lower limit for $\left\langle\tau_{c}\right\rangle$. Clearly it is much larger than $\tau_{l c}$ and the timescales of crystal growth and any possible reorganization are well separated. In contrast, for POM and PEO the timescales of crystallization given by $\tau_{l c}$ and the timescale of reorganization given by the band between $\left\langle\tau_{c}\right\rangle$ and $\tau_{\text {stem }}$ overlap. However, while for POM $\left\langle\tau_{c}\right\rangle$ becomes smaller than $\tau_{l c}$ only for the higher crystallization temperatures above $160{ }^{\circ} \mathrm{C}$,

\footnotetext{
${ }^{1}$ The value given for PEO in ref. ${ }^{20}$ contained an erroneous factor 3.5
} 

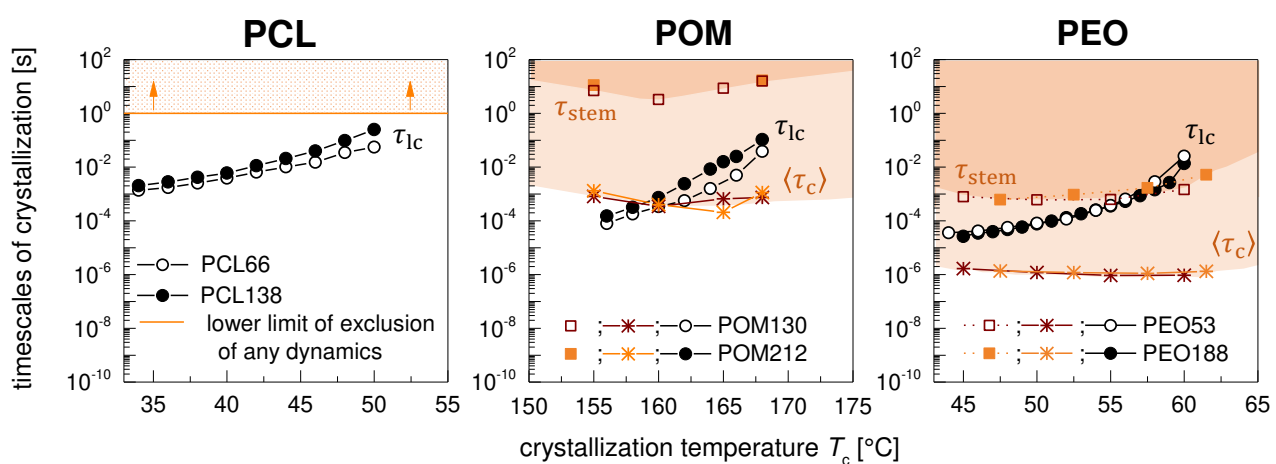

Increasing influence of $\alpha_{c}$-relaxation

Figure 2: Comparison of the timescales of intracrystalline chain dynamics and crystal growth for PCL, POM, and PEO. $\tau_{l c}$ corresponds to the time during which the crystal grows by one molecular layer according to eq.(1). $\left\langle\tau_{c}\right\rangle$ is the average residence time between two helical jumps calculated with eq. (2) and the values given in the SI. $\tau_{\text {stem }}$ denotes the time during which a chain in the crystal diffuses over a distance equal to the lamellar thickness $d_{c}$, estimated by eq.(3). $\left\langle\tau_{c}\right\rangle$ can be considered as lower and $\tau_{\text {stem }}$ as an upper limit of the timescale of crystal reorganization enabled by the $\alpha_{c}$-relaxation. For PCL the solid line shows the NMR detection limit for $\left\langle\tau_{c}\right\rangle$ due to a possibly undetectably slow $\alpha_{c}$-relaxation. ${ }^{35}$

for PEO $\tau_{l c}$ is lying well above $\left\langle\tau_{c}\right\rangle$ for the whole temperature range and even becomes comparable to $\tau_{\text {stem }}$ for the higher crystallization temperatures. Consequently, we confirm that POM fills the relevant gap between PCL and PEO with regard to the ratio between $\tau_{l c}$ and $\left\langle\tau_{c}\right\rangle$, respectively $\tau_{\text {stem }}$, and enables us to establish quantitatively the role of the ICD for crystallization and structure formation. Following the direction of the arrow in Figure 2 from PCL over POM to PEO, we expect an increasing effect of the ICD on crystal growth and the semicrystalline morphology.

\section{Semicrystalline Morphology}

To investigate the effects of ICD on the morphology we performed SAXS measurements during and after isothermal crystallization at different $T_{c}$. Based on a recently refined SAXS analysis $^{36,37}$ we obtain the Porod parameter $P$ as measure of the specific inner surface between crystalline and amorphous regions, the average thicknesses $d_{c / a}$ of the crystalline and amourphous regions together with their distribution widths $\sigma_{c / a}$ in the lamellar stack and 
the long period $L$. Figure 3 shows the results. For each sample system measurements for two

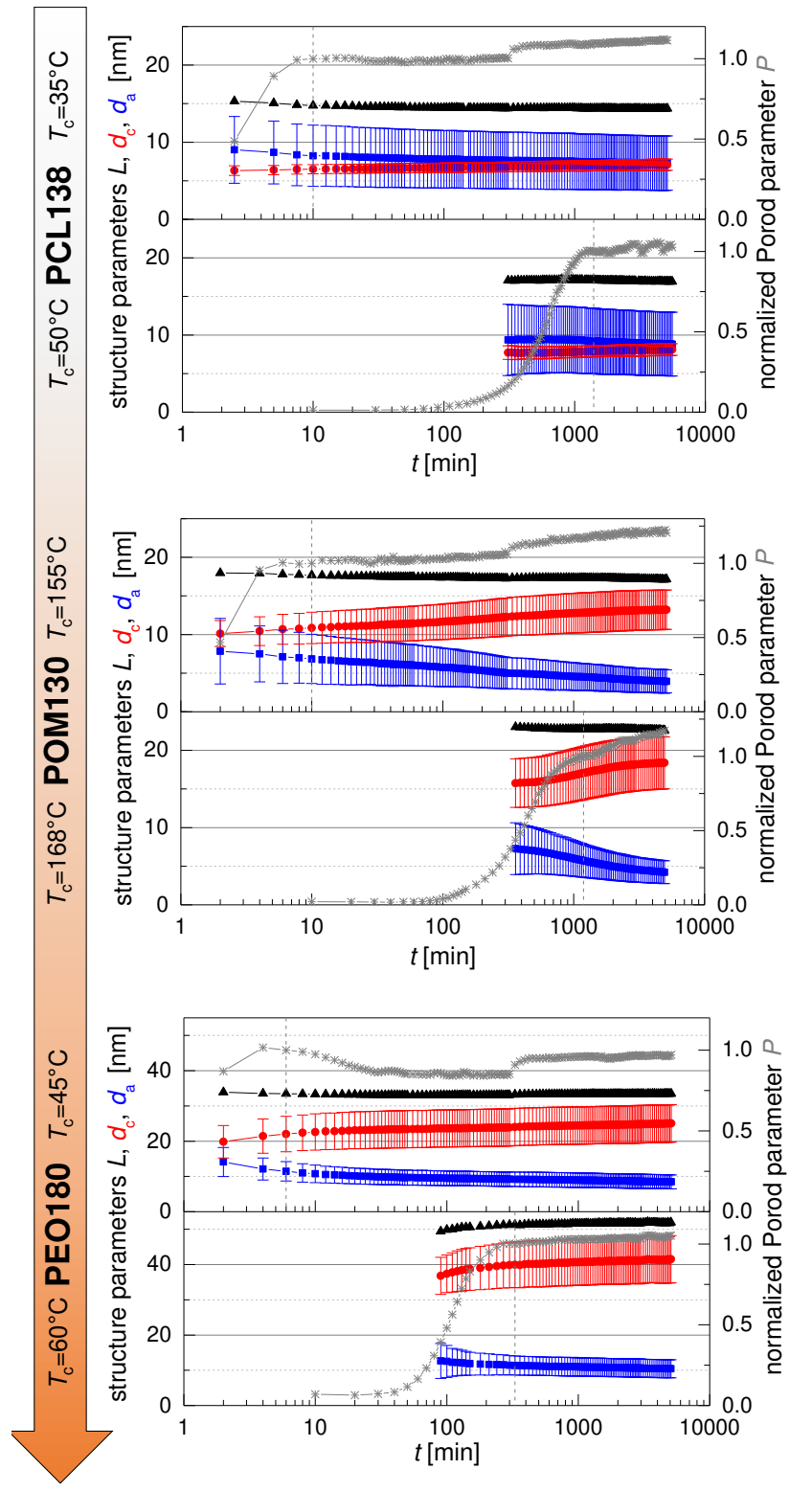

Figure 3: Structure parameters during isothermal crystallization from the melt as a function of the crystallization time obtained by SAXS. For each sample system the isothermal crystallization was performed for a high $(\sim 20 \mathrm{~K})$ and a low $(\sim 5 \mathrm{~K})$ supercooling $\Delta T$. The arrow represents the expected influence of $\alpha_{c}$-relaxation according to Figure 2 . Rhs y-axis: time-dependent Porod parameter $P$ (grey) normalized to 1 at the end of primary crystallization process (dotted line). Lhs y-axis: time-dependent long period $L$ (black), amorphous thickness $d_{a}$ (blue) and crystalline thickness $d_{c}$ (red). $\sigma_{c / a}$ are shown as "error bars". For PEO the scale of the y-axis is increased roughly by factor 2 .

$T_{c} \mathrm{~s}$ are exemplarily shown, corresponding to the lower (supercooling $\Delta T \approx 20 \mathrm{~K}$ ) and the 
upper limit $(\Delta T \approx 5 \mathrm{~K})$ of the experimentally accessible range of isothermal crystallization. The measurements are arranged in such a way that from top to bottom, following the arrow on the left hand side of Figure 3, we expect a growing influence of the ICD. As the Porod parameter $P$ is proportional the amount of crystalline-amorphous interface per volume, we can follow the crystallization process and identify the end of the primary crystallization, which is marked by a vertical, dotted line. For these time-dependent measurements, which go beyond our previous study, we used a PEO sample from a new batch with a slightly lower molecular weight $M_{w}$ than in Figure 2, for PCL and POM the samples are from the same batch as in Figure 2. The sharp steps and oscillations of $P$ during a measurement series are artefacts caused by slight changes in the alignment of the X-ray optics due to remaining temperature variations in the system caused by intermediate closure times of the X-ray shutter and $24 \mathrm{~h}$ temperature oscillations over the day.

PCL shows the already known typical structure of a crystal-fixed polymer with a linear crystallinity around $50 \%$, a well-defined $d_{c}$ (small $\sigma_{c}$ ) and a broader distribution for $d_{a} . d_{c}$ increases only slightly with increasing crystallization temperature. As a new result, observable by the long time series in these measurements, we observe a very small increase in $d_{c}$ and a corresponding decrease in $d_{a}$.

In comparison, POM shows strong structural changes with time for both $T_{c}$. For the lower crystallization temperature these changes mostly take place after the primary crystallization. We observe not only an increase in $d_{c}$ and a decrease in $d_{a}$, but also a decreasing distribution width for $d_{a}$, such that the relative width $\sigma_{a} / d_{a}$ remains approximately constant. With time the morphology develops the typical morphology of a crystal-mobile polymer as observed before in $\mathrm{PEO}^{20,37}$ with a well-defined $d_{a}$ (small $\sigma_{a}$ ) and a more broadly distributed $d_{c}$. Our observations are in keeping with previously observed long time lamellar thickening in $\mathrm{POM}^{38}$, for which we now establish the relatively slow ICD as its origin. The comparison with the higher $T_{c}$ shows that $d_{c}$ depends much more strongly on $T_{c}$ than in case of PCL. Crystal thickening takes place to a large part during primary crystallization and slows down 
afterwards indicating the interplay with crystal growth. Correspondingly, already during primary crystallization a crystal-mobile morphology forms. Generally the linear crystallinity $X_{c}$ is higher than in case of PCL. For $T_{c}=155^{\circ} \mathrm{C} X_{c}$ increases from $65 \%$ at the end of the primary crystallization to $77 \%$ at the last measurement point and from $74 \%$ to $81 \%$ for $T_{c}=168^{\circ} \mathrm{C}$.

In $\mathrm{PEO}$ we observe the typical crystal-mobile structure for both $T_{c}$ already during primary crystallization. In contrast to POM a strong increase/decrease of $d_{c / a}$ can take place already during primary crystallization. Afterwards the changes slow down and also the distributions widths $\sigma_{c / a}$ show no further significant changes. The lamellar thickness $d_{c}$ depends even more strongly on $T_{c}$ than for POM. After finishing the primary crystallization, $X_{c}$ changes from $65 \%$ to $74 \%$ for $T_{c}=45^{\circ} \mathrm{C}$ and from $78 \%$ to $80 \%$ for $T_{c}=60{ }^{\circ} \mathrm{C}$.

The observations for PCL and PEO confirm and extend our previous results. ${ }^{20}$ They correspond to the limiting cases of no or very slow and very fast ICD with the corresponding characteristic morphologies. The results for POM now clearly establish the timescale of ICD and its competition with the one of crystal growth as the relevant effect. We directly observe strong lamellar thickening, which for the higher $T_{c}$ takes place mostly during primary crystallization and for the lower $T_{c}$ after primary crystallization according to the relative values of the characteristic times shown in Figure 2 and represented by the arrow in Figure 3. Important is the observation that crystal thickening slows down around the end of the primary crystallization, which indicates that a certain limiting structure is reached, which is characterized by well-defined minimum value of the amorphous regions, as we concluded already from our previous measurements on PEO. We tentatively explained this limiting structure by a critical entanglement density in the amorphous phase. ${ }^{20,39}$ Here the long-time experiments give interesting new information. The fact that lamellar thickening continues over the whole experimental time scale, shows that this limiting structure is still a nonequilibrium structure and slowly develops further if enabled by ICD. But obviously this process is considerably hindered in a fully developed semicrystalline morphology. On the 
other hand, the observation of longtime lamellar thickening in PCL indicates that even in this, at first sight crystal-fixed polymer, a very slow ICD undetectable by NMR might exist. A further comment concerns the slight decrease of the long period for most of the experiments, which had been observed before for POM and was taken as an indication for insertion crystallization. ${ }^{40}$ Although we cannot completely exclude the existence of such an additional process, we consider it as negligible, as it would be inconsistent with the observed increase of $d_{c}(t)$ and the decrease of $\sigma_{a}(t)$.

The most important new aspect in our data are the quantitative determination of $\left\langle\tau_{c}\right\rangle$ in the relevant temperature range and its evaluation in terms of the kinetics of crystal growth i.e. $\tau_{l c}$, together with the quantitative analysis of the full set of structural parameters. This much broader set of experimental data makes a comparison of the semicrystalline morphology across different polymer systems as in Figure 3 meaningful, whereas typically in the literature the lamellar thickness $d_{c}$ of semicrystalline polymers is discussed only for individual polymer systems in relation to the crystallization temperature $T_{c}$.

A corresponding comparison of the time-dependent value of $d_{c}$ for all $T_{c}$ s and all three polymer systems is shown in Figure 4(A). Additionally to the data already shown in Figure 3, data from further $T_{c}$ s and a second sample (POM212) are included. We observe a systematic increase of $d_{c}$ following the order induced by the ICD indicated as above by the orange arrow. The data suggest, that the value of the crystal thickness is affected from the very beginning of the crystallization by the ICD to a degree which depends on the crystallization temperature as well as on the inherent polymer-dependent timescale of the ICD. The idea is illustrated in the inset of Figure 4(A), which also explains the different curvatures observed for $d_{c}(t)$ for PCL, POM and PEO. In this picture the crystallization process starts with an initial crystalline thickness $d_{c 0}$, which then increases due to reorganization enabled by ICD but is finally restricted by the presence of neighbouring crystallites and the fact that the minimal thickness of the of the amorphous regions is reached. Following this approach, Figure $4(\mathrm{~B})$ shows the largely different values of $d_{c}$ across the three different polymer systems 


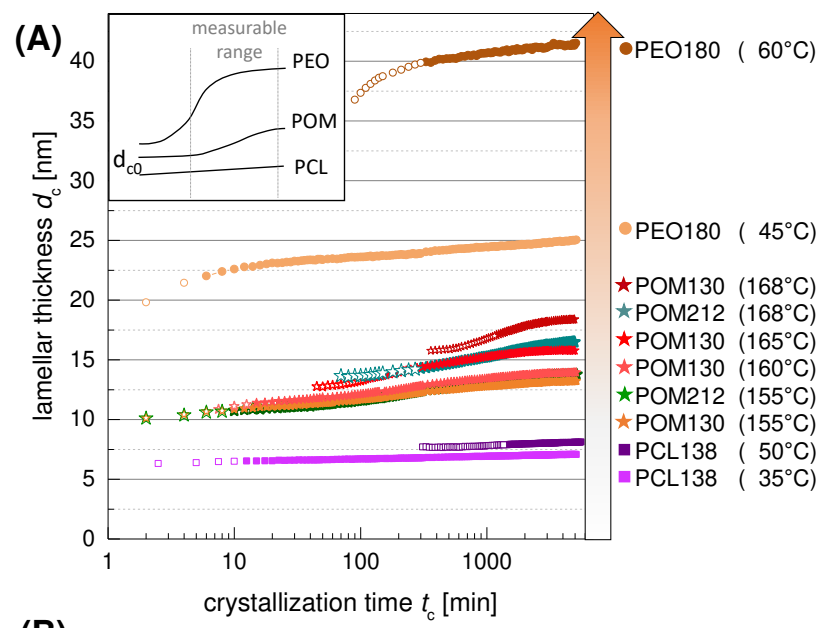

(B)

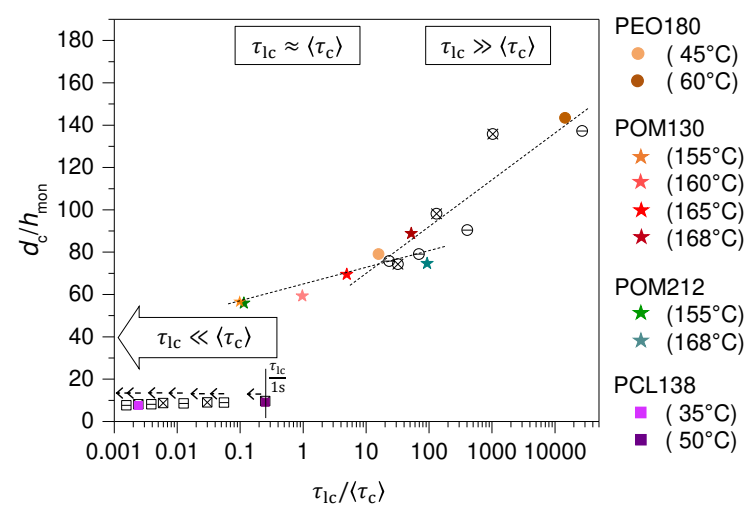

Figure 4: Direct comparison of the crystalline thickness $d_{c}$ for PCL, POM and PEO.(A) $d_{c}$ during isothermal crystallization for all crystallization temperatures. The orange arrow represents the increasing influence of the $\alpha_{c}$-relaxation according to Figure 2 . The change from open to closed symbols indicates the completion of the primary crystallization. (B) Normalized lamellar thickness $d_{c}$ at the end of primary crystallization vs. the ratio of the timescales $\tau_{l c} /\left\langle\tau_{c}\right\rangle$. Depending on the ratio $\tau_{l c} /\left\langle\tau_{c}\right\rangle$ different regimes are visible. The dotted lines are guides to the eye. The closed symbols are the data from Figure 4(A), open symbols represent already published data of PEO (open circles) and PCL (open squares) for two molecular weights (cross and line) measured at different $T_{c} \cdot{ }^{20}$ For PCL a constant value of $1 \mathrm{~s}$ was taken for $\left\langle\tau_{c}\right\rangle$, corresponding to the lower limit of a possible $\alpha_{c}$-relaxation time and an upper limit of $\tau_{l c} /\left\langle\tau_{c}\right\rangle$ as indicated by the broken arrow.

as a function of the ratio of the characteristic times $\left\langle\tau_{c}\right\rangle / \tau_{l c}$, which depends on the polymer system as well as on the crystallization temperature. Additionally, $d_{c}$ is normalized by the height of a monomer unit in direction of the stem, as this is the distance over which the (local part) of the chain moves during one helical jump ${ }^{16}$, neglecting a possible chain tilt. The factors $h_{m o n}$ were calculated from the length of the unit cell in $c$-direction (PEO: 
$\frac{1.95}{7} \mathrm{~nm}^{33}$; POM: $\frac{1.73}{9} \mathrm{~nm}^{34}$; PCL: $\left.\frac{1.73}{2} \mathrm{~nm}^{41}\right)$. A reorganisation on the timescale of the primary crystallization $\left(\tau_{l c} \gg\left\langle\tau_{c}\right\rangle\right)$, as for $\mathrm{PEO}$, leads to high $d_{c}$ values. A reorganisation, which is slower in comparison to crystal growth as for $\mathrm{POM}\left(\tau_{l c} \approx\left\langle\tau_{c}\right\rangle\right)$, leads to smaller values and is eventually more restricted by neighbouring crystallites. Accordingly, there is a more or less smooth progression of the $d_{c}$-values from POM to PEO, whereas the values for PCL fall out of trend in line with the fact, that PCL has no or very slow ICD $\left(\tau_{l c} \ll\left\langle\tau_{c}\right\rangle\right)$. Reorganization plays no role during primary crystallization and the crystallites keep basically the initial $d_{c 0}$.

Combining SAXS with ultrafast scanning calorimetry we recently showed that in this latter case of PCL the crystalline lamellae are only marginally stable, i.e. they melt immediately upon heating and $T_{m} \approx T_{c} \cdot{ }^{20,21}$ In this case, the crystals grow obviously with very small supercooling and the initial crystal thickness is basically controlled by thermodynamics. The corresponding effect of temperature is weak, as the measurements shown here for $T_{c}=35^{\circ} \mathrm{C}$ and $T_{c}=50^{\circ} \mathrm{C}$ illustrate.

\section{Discussion}

How do these observations compare with previous experimental results? Lamellar thickening as a fundamental process occuring in semicrystalline polymers is well known. ${ }^{42}$ However, direct observations including the regime of primary crystallization has been scarce. ${ }^{43}$ Common are either DSC observations, where an increase of the melting temperature is interpreted as an indication for lamellar thickening, or SAXS measurements of the long period. ${ }^{44,45}$ Long-time annealing experiments gave evidence for the logarithmic time dependence also oberved here at times beyond the primary crystallization. ${ }^{46}$ Nearly all experiments deal with polyethylene, a common crystal-mobile polymer, which however has the disadvantage that it is difficult to measure $\left\langle\tau_{c}\right\rangle$ by NMR and also samples with well-defined molecular weight and low polydispersity are difficult to synthesize. A long standing discussion concerned the question if on a microscopic level chain movement is caused by a sliding motion 
of the whole chain or induced local defects. ${ }^{47}$ Atomistic simulations performed for the case of PE showed that a number of different localized confomational defects cause ICD, a result consistent with the weak thickness dependence and broad distribution of $\left\langle\tau_{c}\right\rangle$ observed in our experimental results on PEO and POM. ${ }^{48}$

Going beyond these existing observations we suggest, that generally the large differences in $d_{c}$ found between different polymer systems and for different $T_{c}$ in case of crystal-mobile polymers are caused by a kinetically controlled thickening process based on crystal reorganisation due to ICD taking place practically simultaneously with crystal growth. In accordance to this scenario, the values of the crystal thickness reported in the literature for other crystalfixed polymers ${ }^{16}$ are similar as observed here for PCL $\left(\mathrm{PET}^{49}: d_{c} \sim 3 \mathrm{~nm}\right.$ to $6 \mathrm{~nm}, \mathrm{PBT}^{50,51}$ : $d_{c} \sim 6 \mathrm{~nm}$ to $7 \mathrm{~nm}, \mathrm{sPP}^{52}: d_{c} \sim 7 \mathrm{~nm}$ to $\left.8 \mathrm{~nm}\right)$. Also in these cases time-resolved SAXS shows no lamellar thickening. ${ }^{49,50,52} \mathrm{PE}$ on the other hand, is a well studied crystal-mobile polymer; lamellar thickening is reported ${ }^{53-55}$ and $T_{c}$ dependent values in the range of $d_{c} \sim 20 \mathrm{~nm}$ to $60 \mathrm{~nm}^{56}$ suggest, that PE behaves similar to PEO. Different NMR-studies have indeed verified a comparably fast ICD in this polymer. ${ }^{57-60}$ In this scenario, crystallization of PE in the hexagonal high pressure phase that leads to crystal thicknesses on the micrometre scale, would be the extreme case. Compared to the orthorhombic phase occuring under normal conditions, the ICD in this phase is about three orders of magnitude faster going along with a high conformational and partially positional disorder. ${ }^{61,62}$

In conclusion, the experiments on poly(oxymethylene) confirm our hypothesis that the morphology of semicrystalline polymers results from an interplay or competition of crystal growth and crystal thickening due to intracrystalline chain dynamics (ICD). NMR measurements confirmed that in this polymer the ICD takes place on an intermediate timescale. The results complement our previous experiments on PCL and PEO, which correspond to the cases where the ICD is either very slow or fast in comparison to crystal growth. Across all three polymer systems we observed for a first time a systematic increase of the lamellar thickness, which for POM and PEO follows smoothly the increasing ratio of the characteristic 
times $\tau_{l c} /\left\langle\tau_{c}\right\rangle$. These findings explain on the one hand the large differences in crystal thickness of different sample systems, on the other hand the well established strong dependence of $d_{c}$ on the crystallization temperature for many common polymers, which are crystal-mobile. Thus, a unifying picture of the crystallization process emerges, in which crystallization starts with an initial thin crystal, whose thickness corresponds to marginal thermodynamic stability and continues with a kinetically controlled lamellar thickening, resulting in a further thermodynamic stabilization. The thickening is enabled by the existence of ICD, which is a typical property of polymer crystals.

An important observation is the fact that the initially fast thickening of POM at high $T_{c}$ strongly slowed down during crystallization ending up in the known very slow logarithmic growth. This fact together with the observation, that reorganization always led to a well-defined, narrowly distributed amorphous thickness, indicates that crystal thickening is increasingly restrained or suppressed by constraints in the amorphous regions, namely the entanglements. The crystal thickness observed in a fully crystallized sample is therefore strongly dependent on the ratio $\tau_{l c} /\left\langle\tau_{c}\right\rangle$, i.e. on the time available for more or less unhindered reorganization. Previous explanations based on an assumed significant slowing down of the ICD due to increasing crystal thickness are inconsistent with our experimental result, that $\left\langle\tau_{c}\right\rangle$ is either very weakly dependent on $d_{c}(\mathrm{PEO})$ or independent of $d_{c}(\mathrm{POM})$.

Generally our results highlight the fact that the semicrystalline morphology is a nonequilibrium structure and to a large extent controlled by reorganization. Specifically for crystal-mobile polymers it will in general not be possible to separate crystal growth and crystal reorganization, which makes the observation of the initial crystal thickness difficult if not impossible. Theoretical predictions for the crystal thickness, which do not take into account crystal thickening, can therefore not be applied to crystal-mobile polymers, although it has often been done in the past. Furthermore it is important to not only consider the crystal thickness but also the thickness of the amorphous regions, which is the better defined parameter for crystal-mobile polymers. It seems that, opposite to existing views, it is the 
internal structure of the amorphous regions which limits the crystal thickness and therefore the crystallinity of crystal-mobile polymers. Crystal-fixed polymers without or with very slow ICD allow to determine the crystal thickness as a function of supercooling, but the dependence is much weaker than for crystal-mobile polymers. The crystals in these systems display only a marginal thermodynamic stability directly after crystallization, which takes away one of the main observations taken as evidence for a postulated intermediate mesophase, whose stability with respect to the crystal phase was assumed to determine the crystal thickness. On the other hand, in line with the multistage models reorganization plays a large role in polymer crystallization, but in the model systems we investigated it takes place in the crystal phase itself and is based on intracrystalline chain dynamics (ICD). While we could already show in a previous publication that the selected thickness of the amorphous regions in crystal-fixed polymers goes along with an increase of the entanglement concentration by about a factor of two, ${ }^{39}$ a more detailed investigation of entanglement effects on the morphology especially for crystal-mobile polymers is still lacking. From our results one would expect that entanglements are partially dissolved during thickening by ICD, a prediction which would be interesting to demonstrate directly in future. Such studies would also take up results from simulations ${ }^{63}$ and analytical theoretical work ${ }^{64}$, in which the role of entanglements is considered.

\section{Methods}

\section{Materials}

As model systems we chose poly(- $\epsilon$-caprolactone) (PCL), poly(oxymethylene) (POM) and poly(ethyleneoxide) (PEO). PEO with a very fast and PCL without or very slow $\alpha_{c}$-relaxation ${ }^{16,32,35}$ were already investigated in detail in previous publications. ${ }^{20,21} \mathrm{POM}$ shows a comparably slow $\alpha_{c}$-relaxation. ${ }^{16}$ The sample characteristics are given in Table 1. For each sample system we investigated two different molecular weights in order to exclude any spe- 
cial molecular weight effects and to show the generality of the results. The samples were named after the molecular weight $M_{\mathrm{w}}$, which was determined by GPC-analysis. For PCL a polystyrene calibration and THF as solvent was used. For PEO a polystyrene calibration and $\mathrm{H}_{2} \mathrm{O}$ with $0.5 \mathrm{~g} / \mathrm{l} \mathrm{NaN3}$ as solvent was used. The poly(oxymethylenes) are industrial samples, containing a not further specified amount of stabilizers to avoid degradation by the mechanism observed by Kern and Stohler. ${ }^{67,68}$ The molecular weight was determined using poly(methylmethacrylate) calibration and HFIP/ $0.05 \mathrm{M} \mathrm{KTFAC} \mathrm{as} \mathrm{solvent.} \mathrm{All} \mathrm{polymers}$ have a molecular weight $M_{\mathrm{w}}$ well above the entanglement molecular weight $M_{\mathrm{e}}$ and the contour length $R_{\max }$ is much larger than the typical size of the semicrystalline structure. Hence, the chosen polymers are representative for crystallization from an entangled polymer melt. Melting temperatures $T_{m}$ and crystallinity $X_{c}$ given in Table 1 were determined by DSC; $X_{c}=\Delta H / \Delta H_{100}$. Here, $\Delta H$ is the measured melting enthalpy and $\Delta H_{100}$ the extrapolated melting enthalpy for a $100 \%$ crystalline sample (POM: $\Delta H_{100}=326 \mathrm{~J} / \mathrm{g}^{69}$; PCL: $\Delta H_{100}=157 \mathrm{~J} / \mathrm{g}^{70} ;$ PEO: $\left.\Delta H_{100}=196.6 \mathrm{~J} / \mathrm{g}^{71}\right)$. As POM is sensitive for degradation at high temperatures, the isothermal crystallization step during sample preparation was performed either under nitrogen atmosphere (NMR and DSC) or under vacuum (SAXS).

Table 1: Sample characteristics. The molecular weights and therefore also $R_{\max }$ are nominal values based on the GPC calibrations given in the text. Only for PCL a correction factor of 0.56 is known, which would reduce $R_{\max }$ to a value of $413 \mathrm{~nm} .{ }^{66}$ The melting temperatures (peak maxima) and crystallinities were obtained by DSC heating scans. The samples were cooled from the melt and heated with a rate of $10 \mathrm{~K} / \mathrm{min}$.

\begin{tabular}{|c|c|c|c|c|c|c|c|}
\hline Sample & supplier/ industry name & $\begin{array}{c}M_{\mathrm{w}} \\
{[\mathrm{kg} / \mathrm{mol}]}\end{array}$ & $\begin{array}{c}M_{\mathrm{n}} \\
{[\mathrm{kg} / \mathrm{mol}]}\end{array}$ & $\begin{array}{c}R_{\max } \\
{[\mathrm{nm}]}\end{array}$ & $\begin{array}{c}M_{\mathrm{e}} \\
{[\mathrm{kg} / \mathrm{mol}]}\end{array}$ & $\begin{array}{c}T_{\mathrm{m}} \\
{\left[{ }^{\circ} \mathrm{C}\right]}\end{array}$ & $\begin{array}{c}X_{c} \\
{[\%]}\end{array}$ \\
\hline \hline POM130 & DuPont/ Delrin ${ }^{\circledR}$ 500P NC010 & 130 & 39.9 & 255 & $2.644^{65}$ & 177 & 61 \\
POM212 & DuPont/ Delrin ${ }^{\circledR}$ 100P NC010 & 212 & 62.8 & 402 & & 179 & 59 \\
\hline PCL138 & Sc. Polym. Products, Inc./- & 138 & 97.4 & 738 & $2.5 \pm 0.5^{39}$ & 58 & 40 \\
\hline PEO180 & PSS Polymer Standards Service/- & 180 & 148 & 936 & $2.00^{65}$ & 69 & 74 \\
\hline
\end{tabular}




\section{Instruments and data analysis}

\section{Small angle X-ray scattering}

SAXS measurements were performed on a Kratky compact camera from AntonPaar GmbH equipped with a focusing X-ray optics from AXO Dresden GmbH and with a 1D detector Mythen2 R 1K from Dectris. A temperature-controlled sample holder enabled in-situ isothermal crystallization experiments at different crystallization temperatures $T_{c}$.

The data were analyzed using a quantitative approach based on modeling the interface distribution function. A short account of the method is given in the SI, further details can be found in in refs. ${ }^{20,36}$. The analysis provides the mean thickenesses of the crystalline $\left(d_{c}\right)$ and amorphous $\left(d_{a}\right)$ domains together with their distribution widths $\sigma_{c}$ and $\sigma_{a}$ in terms of assumed Gaussian distributions as well the Porod parameter $P$. The width of the window function used to smooth the interface distribution functions was $1.0 \mathrm{~nm}$ for PCL, $0.8 \mathrm{~nm}$ for POM and $1.4 \mathrm{~nm}$ for PEO.

\section{${ }^{13}$ C MAS CODEX}

Rotor-synchronized CODEX (centerband-only detection of exchange) experiments were performed on a $400 \mathrm{MHz}$ Bruker Avance system with a ${ }^{13} \mathrm{C}$ Larmor frequency of $100.6 \mathrm{MHz}$ using double and triple resonance probes at a spinning rate of $5000 \pm 3 \mathrm{~Hz}$. During the evolution and the acquisition of the ${ }^{13} \mathrm{C}$ signal high power proton decoupling (SPINAL64) was used. The $\pi / 2$-pulses of the ${ }^{1} \mathrm{H}$ and ${ }^{13} \mathrm{C}$ were set to 3.0 and $3.3 \mu \mathrm{s}$, respectively. The recoupled evolution time $N \tau_{R}$ was set to $1.2 \mathrm{~ms}$ with a MAS rotor period $\tau_{R}=200 \mu \mathrm{s}$ and $N$ as an even integer number. The recycle delay $d_{1}$ (time between successive scans) and cross polarization (CP) time were $8-16 \mathrm{~s}$ and $900 \mu \mathrm{s}$, respectively.

The ${ }^{13} \mathrm{C}$ MAS CODEX technique ${ }^{31}$ probes slow reorientations of the ${ }^{13} \mathrm{C}$ chemical shift anisotropy (CSA) tensor and has often been used to investigate slow dynamics in semicrys- 


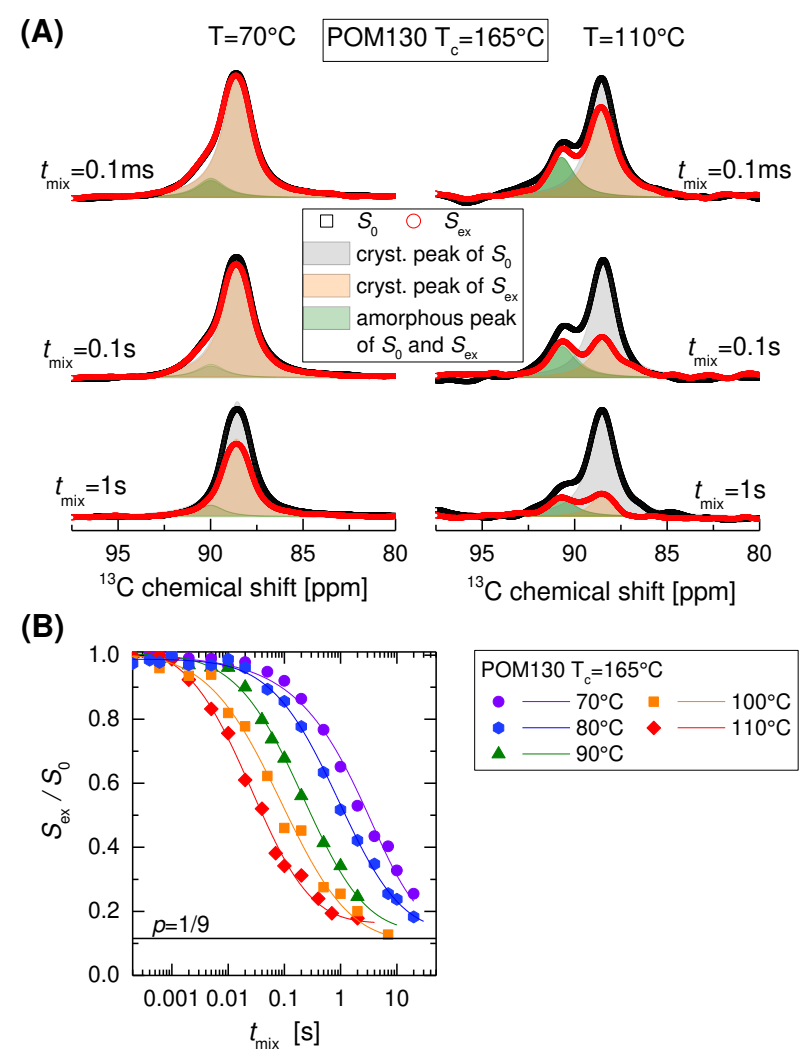

Figure 5: Exemplary NMR analysis on the POM samples. (A) ${ }^{13} \mathrm{C}$ CP MAS spectra of the exchange $S_{e x}$ (red) and reference signal $S_{0}$ (black) for three different mixing times at two temperatures. The resonances are deconvoluted into the amorphous (green) and crystalline exchange (orange) and reference (gray) peak. (B) CODEX $t_{m i x}$ decay curves for four different temperatures resulting in the Arrhenius temperature dependence shown in Figure 1(C).

talline polymers. ${ }^{32}$ In the CODEX experiment ${ }^{31}$ the CSA is refocused by rotor-synchronized $\pi$-pulses during an evolution and a reconversion period which are separated by a mixing time $t_{m i x}$. If no reorientation of the CSA tensor occurs during the mixing time, the signal will be completely recoupled. Molecular motions during the $t_{m i x}$ lead to a signal decay of the exchange intensity $S_{e x}$. A reference signal $S_{0}$ is acquired with a short $t_{m i x}$ to compensate for signal losses caused by relaxation effects. In Figure 5(A) the effect of the mixing time on the exchange and reference signal is shown. The crystalline exchange signal is reduced by relaxation effects and signal losses caused by the reorientation of the CSA tensor, the difference between reference and exchange signal increases for longer $t_{m i x}$ and higher temperatures (faster intracrystalline dynamics). The area under the amorphous peak (green area in 
Figure 5(A)) is similar for both signals $S_{e x}$ and $S_{0}$ and is only affected by relaxation effects. To analyse the correlation time $\left\langle\tau_{c}\right\rangle$ describing the intracrystalline dynamics the signal decay $S_{e x} / S_{0}$ is fitted via

$$
S_{e x}\left(t_{m i x}\right) / S_{0}\left(t_{m i x}\right)=p+(1-p) \cdot \exp \left[-\left(t_{m i x} / \tau_{c}\right)\right]
$$

with $p=1 / M$ with $M$ distinguishable sites $\left(M=9\right.$ for the $9_{5}$-helix in POM) as shown in Figure 5 (B). Additionally we assume a lognormal distribution of the helical jump correlation time. The distribution width $\sigma$ attains values between 1.3 and 2.0, corresponging to a distribution extending over 1-2 decades. With regards to possible contributions from spin diffusion, which is largely temperature-independent and very slow but measurable for ${ }^{13} \mathrm{C}$ at natural abundance, we found apparent, possibly spin-diffusion dominated values for $\tau_{\mathrm{c}}$ of order 100s and above at temperatures of $30^{\circ} \mathrm{C}$ and below. These lower-limit estimates are one order of magnitude larger than $\tau_{\mathrm{c}}$ in the temperature range of interest at $T>60^{\circ} \mathrm{C}$. Therefore, we can safely ignore spin diffusion. See also the SI (Figure S1).

\section{Polarisation microscopy}

Polarization microscopy experiments were performed on an Olympus BX51 microscope equipped with a Linkam hot stage THMS600, temperature controler TP94 and liquid nitrogen controler LNP. Samples were held between two glass slides and had a thickness of several $10 \mu \mathrm{m}$ up to $80 \mu \mathrm{m}$. After fast cooling from the melt to different crystallization temperatures $T_{c}$, a series of images was recorded during isothermal crystallization and the growth velocity of spherulites was determined from their time dependent area. For every temperature an average over 3 different spherulites was performed. 
DSC

DSC measurements were performed with a UNIX DSC 7 from Perkin Elmer. Nitrogen was used as a purging gas, temperature calibration was performed with mercury.

\section{Appendix}

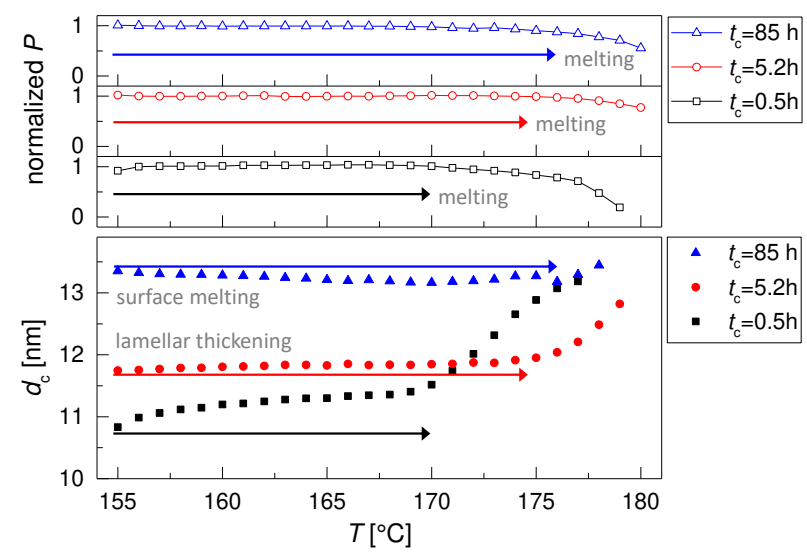

Figure 6: Effect of crystallization time on melting temperature. Porod parameter $P$ and crystal thickness $d_{c}$ of POM130 during stepwise heating after isothermal crystallization at $155^{\circ} \mathrm{C}$ for different crystallization times: $t_{c}=0.5 \mathrm{~h}$ (black), $t_{c}=5.2 \mathrm{~h}$ (red) and $t_{c}=85 \mathrm{~h}$ (blue)

Thickening should always lead to further thermodynamic stabilization and an increased melting temperature. We observed such effects previously in $\mathrm{PEO}^{20}$, however for $\mathrm{POM}$ it can be demonstrated directly as we follow the thickening process at the higher crystallization temperature. Figure 6 shows the Porod parameter $P$ and lamellar thickness $d_{c}$ as resulting from in-situ SAXS experiments during stepwise heating after isothermal crystallization at $T_{c}=155^{\circ} \mathrm{C}$ for different crystallization times $t_{c}$. Generally, the melting process goes along with a strong decrease of $P$ and an increase of the average value $d_{c}$, due to the melting of thinner lamellae. The dominating processes affecting $d_{c}$ during heating before final melting are indicated in Figure 6. Indeed with increasing crystallization time $t_{c}$ the the melting process shifts to higher temperatures. For crystallization times of $0.5 \mathrm{~h}$ and $5.2 \mathrm{~h}$ the lamellar thickening process continues also during heating. Only for the longest crystallization time 
of $85 \mathrm{~h}(30600 \mathrm{~min})$ the trend is inverted. Now heating leads to a decrease of $d_{c}$ in the temperature range below final melting, an effect well-known for $\mathrm{PE}^{72}$ and $\mathrm{PEO}^{20}$, which is called surface melting and caused by a local equilibrium between the crystals and the adjacent amorphous phase. These observations are in keeping with the hypothesis that crystal thickening is restricted by the contraints in the amorphous phase.

\section{Data availability}

All data needed to evaluate the conclusions in the paper are present in the paper and/or the Supplementary information. The data shown in Fig. 3 and Fig. 4a are available in digital format in the Supplementary information. Further datasets generated during the current study are available from the corresponding authors on reasonable request.

\section{References}

(1) Strobl, G. The physics of polymers. Concepts for understanding their structures and behavior, 3rd ed.; Springer: Berlin, 2007; DOI: 10.1007/978-3-540-68411-4.

(2) Tang, X.; Chen, W.; Li, L. The Tough Journey of Polymer Crystallization: Battling with Chain Flexibility and Connectivity. Macromolecules 2019, 52, 3575-3591, DOI: 10.1021/acs.macromol.8b02725.

(3) Jiang, X.; Reiter, G.; Hu, W. How Chain-Folding Crystal Growth Determines the Thermodynamic Stability of Polymer Crystals. Journal of Physical Chemistry B 2016, 120, 566-571, DOI: 10.1021/acs.jpcb.5b09324.

(4) Hoffman, J.; Lauritzen, J. Crystallization of Bulk Polymers with Chain Folding: Theory of Growth of Lamellar Spherulites. Journal of Research of the National Bureau of Standards 1961, A 65, 297-336, DOI: 10.6028/jres.065A.035.

(5) Gedde, U. W. Polymer physics, 1st ed.; Chapman and Hall: London, 1995.

(6) Sadler, D.; Gilmer, G. Rate-Theory Model of Polymer Crystallization. Physical Review Letters 1986, 56, 2708-2711, DOI: 10.1103/PhysRevLett.56.2708.

(7) Sadler, D. M. New explanation for chain folding in polymers. Nature 1987, 326, 174177, DOI: $10.1038 / 326174 a 0$.

(8) Stepanow, S. Kinetic mechanism of chain folding in polymer crystallization. Physical Review e 2014, 90, 032601, DOI: 10.1103/PhysRevE.90.032601. 
(9) Keller, A.; Hikosaka, M.; Rastogi, S.; Toda, A.; Barham, P.; Goldbeck-Wood, G. An Approach to the Formation and Growth of New Phases with Application to Polymer Crystallization - Effect of Finite-Size, Metastability, and Ostwald Rule of Stages. Journal of Materials Science 1994, 29, 2579-2604, DOI: 10.1007/BF00356806.

(10) Strobl, G. From the melt via mesomorphic and granular crystalline layers to lamellar crystallites: A major route followed in polymer crystallization? European Physical Journal e 2000, 3, 165-183, DOI: 10.1007/s101890070030.

(11) Strobl, G. Colloquium: Laws controlling crystallization and melting in bulk polymers. Reviews of Modern Physics 2009, 81, 1287-1300, DOI: 10.1103/RevModPhys.81.1287.

(12) Robelin-Souffache, E.; Rault, J. Origin of the Long Period and Crystallinity in Quenched Semicrystalline Polymers 1. Macromolecules 1989, 22, 3581-3594.

(13) Hikosaka, M. Unified theory of nucleation of folded-chain crystals and extendedchain crystals of linear-chain polymers. Polymer 1987, 28, 1257-1264, DOI: 10.1016/0032-3861 (87) 90434-4.

(14) Boyd, R. H. Relaxation processes in crystalline polymers: Molecular interpretation - a review. Polymer 1985, 26, 1123-1133, DOI: 10.1016/0032-3861 (85) 90240-X.

(15) Boyd, R. H. Relaxation processes in crystalline polymers: experimental behaviour - a review. Polymer 1985, 26, 323-347, DOI: 10.1016/0032-3861(85)90192-2.

(16) Hu, W.; Schmidt-Rohr, K. Polymer ultradrawability: the crucial role of alpharelaxation chain mobility in the crystallites. Acta Polymerica 1999, 50, 271-285, DOI: 10.1002/(SICI) 1521-4044(19990801) $50: 8<271:$ : AID-APOL271>3 . 0 . CO ; 2-Y.

(17) Miyoshi, T.; Mamun, A.; Reichert, D. Fast Dynamics and Conformations of Polymer in a Conformational Disordered Crystal Characterized by ${ }^{1} \mathrm{H}-{ }^{13} \mathrm{C}$ WISE NMR. Macromolecules 2010, 43, 3986-3989, DOI: 10.1021/ma901927m.

(18) Miyoshi, T.; Mamun, A. Critical roles of molecular dynamics in the superior mechanical properties of isotactic-poly(1-butene) elucidated by solid-state NMR. Polymer Journal 2012, 44, 65-71, DOI: 10.1038/pj.2011.66.

(19) Hong, Y. L.; Koga, T.; Miyoshi, T. Chain Trajectory and Crystallization Mechanism of a Semicrystalline Polymer in Melt- and Solution-Grown Crystals As Studied Using C-13-C-13 Double-Quantum NMR. Macromolecules 2015, 48, 3282-3293, DOI: 10.1021/acs.macromol.5b00079.

(20) Schulz, M.; Seidlitz, A.; Kurz, R.; Bärenwald, R.; Petzold, A.; Saalwächter, K.; ThurnAlbrecht, T. The Underestimated Effect of Intracrystalline Chain Dynamics on the Morphology and Stability of Semicrystalline Polymers. Macromolecules 2018, 51, 83778385, DOI: 10.1021/acs.macromol.8b01102. 
(21) Schulz, M.; Seidlitz, A.; Petzold, A.; Thurn-Albrecht, T. The effect of intracrystalline chain dynamics on melting and reorganization during heating in semicrystalline polymers. Polymer 2020, 196, 122441, DOI: 10.1016/j . polymer.2020.122441.

(22) Ishida, Y.; Matsuo, M.; Ito, H.; Yoshino, M.; Irie, F.; Takayanagi, M. Dielectric behavior and visco-elastic behavior of polyoxymethylene (Delrin). Kolloid-Zeitschrift 1961, 174, 162-163, DOI: $10.1007 / B F 01559381$.

(23) McCrum, N. G. Internal friction in polyoxymethylene. Journal of Polymer Science 1961, 54, 561-568, DOI: 10.1002/pol.1961.1205416019.

(24) Read, B.; Williams, G. The Dielectric and Dynamic Mechanical Properties of Polyoxymethylene (Delrin). Polymer 1961, 2, 239-255, DOI: 10.1016/0032-3861(61)90028-3.

(25) Arisawa, K.; Tsuge, K.; Wada, Y. Dielectric Relaxations in Polyoxymethylene and Polyethylene Oxide. Japanese Journal of Applied Physics 1965, 4, 138-147, DOI: 10.1143/JJAP. 4.138.

(26) Miki, K.; Hikichi, K.; Kaneko, M. Dynamic Mechanical Properties of Polyoxymethylene .2. Japanese Journal of Applied Physics 1967, 6, 931-937.

(27) Gray, R. On the $\alpha$-Relaxation in Bulk Polyoxymethylene. Journal of Materials Science 1973, 8, 1673-1689, DOI: 10.1007/BF02403516.

(28) Schmidt-Rohr, K.; Spiess, H. W. Multidimensional solid-state NMR and polymers; Acad. Press: London, 1994.

(29) Kentgens, A. P. M.; de Boer, E.; Veeman, W. S. Ultraslow molecular motions in crystalline polyoxymethylene. A complete elucidation using two-dimensional solid state NMR. The Journal of Chemical Physics 1987, 87, 6859-6866, DOI: $10.1063 / 1.453730$.

(30) Karahaliou, P.; Kerasidou, A.; Georga, S.; Psarras, G.; Krontiras, C.; KargerKocsis, J. Dielectric relaxations in polyoxymethylene and in related nanocomposites: Identification and molecular dynamics. Polymer 2014, 55, 6819-6826, DOI: $10.1016 / j$. polymer . 2014.10.056.

(31) DeAzevedo, E.; HU, W.; Bonagamba, T.; Schmidt-Rohr, K. Centerband-only Detection of Exchange: Efficient Analysis of Dynamics in Solids by NMR. J. Am. Chem. Soc 1999, 121, 8411-8412, DOI: 10.1021/ja992022v.

(32) Kurz, R.; Achilles, A.; Chen, W.; Schäfer, M.; Seidlitz, A.; Golitsyn, Y.; Kressler, J.; Paul, W.; Hempel, G.; Miyoshi, T.; Thurn-Albrecht, T.; Saalwächter, K. Intracrystalline Jump Motion in Poly(ethylene oxide) Lamellae of Variable Thickness: A Comparison of NMR Methods. Macromolecules 2017, 50, 3890-3902, DOI: 10.1021/acs.macromol.7b00843. 
(33) Takahashi, Y.; Tadokoro, H. Structural Studies of Polyethers, (-(CH2)m-O-)n X. Crystal Structure of Poly(ethylene oxide). Macromolecules 1973, 6, 672-675.

(34) Tadokoro, H.; Chatani, Y.; Yoshihara, T.; Tahara, S.; Murahashi, S. Structural studies on polyethers, [-(CH2)m-O-]n. II. Molecular structure of polyethylene oxide. Die Makromolekulare Chemie 1964, 73, 109-127, DOI: 10.1002/macp.1964.020730109.

(35) Schäler, K.; Achilles, A.; Bärenwald, R.; Hackel, C.; Saalwächter, K. Dynamics in Crystallites of Poly(epsilon-caprolactone) As Investigated by Solid-State NMR. Macromolecules 2013, 46, 7818-7825, DOI: 10.1021/ma401532v.

(36) Seidlitz, A.; Thurn-Albrecht, T. In Polymer Morphology; Guo, Q., Ed.; 2016; pp 151164, DOI: $10.1002 / 9781118892756 . c h 9$.

(37) Qiao, Y.; Schulz, M.; Wang, H.; Chen, R.; Schäfer, M.; Thurn-Albrecht, T.; Men, Y. Hierarchical structure of polybutene-1 in crystal blocks resulting from the form II to I solid-to-solid transition as revealed by small-angle X-ray scattering. Polymer 2020, 195, 122425, DOI: 10.1016/j.polymer.2020.122425.

(38) Sauer, B.; Mclean, R.; Londono, J.; Hsiao, B. Morphological changes during crystallization and melting of polyoxymethylene studied by synchrotron X-ray scattering and modulated differential scanning calorimetry. Journal of Macromolecular SciencePhysics 2000, B39, 519-543, DOI: 10.1081/MB-100100402.

(39) Kurz, R.; Schulz, M.; Scheliga, F.; Men, Y.; Seidlitz, A.; Thurn-Albrecht, T.; Saalwächter, K. Interplay between Crystallization and Entanglements in the Amorphous Phase of the Crystal-Fixed Polymer Poly( $\epsilon$-caprolactone). Macromolecules 2018, 51, 5831-5841, DOI: 10.1021/acs .macromol.8b00809.

(40) Hama, H.; Tashiro, K. Structural changes in isothermal crystallization process of polyoxymethylene investigated by time-resolved FTIR, SAXS and WAXS measurements. Polymer 2003, 44, 6973-6988, DOI: 10.1016/j . polymer.2003.08.019.

(41) Bittiger, H.; Marchess, R. H.; Niegisch, W. D. Crystal Structure of Poly-EtaCaprolactone. Acta Crystallographica Section B-Structural Crystallography and Crystal Chemistry 1970, B 26, 1923-1927, DOI: Doi $\sqcup 10.1107 /$ S0567740870005198.

(42) Miyoshi, T.; Mamun, A.; Hu, W. Molecular Ordering and Molecular Dynamics in Isotactic-Polypropylene Characterized by Solid State NMR. The Journal of Physical Chemistry B 2010, 114, 92-100, DOI: 10.1021/jp908649y.

(43) Albrecht, T.; Strobl, G. Observation of the early stages of crystallization in polyethylene by time-dependent SAXS: Transition from individual crystals to stacks of lamellae. Macromolecules 1996, 29, 783-785, DOI: 10.1021/ma9503524.

(44) Weeks, J. J. Melting Temperature and Change of Lamellar Thickness with Time for Bulk Polyethylene. J Res Natl Bur Stand A Phys Chem 1963, 67A, 441-451, DOI: 10.6028/jres.067A.046. 
(45) Barham, P.; Keller, A. The Initial-Stages of Crystallization of Polyethylene from the Melt. Journal of Polymer Science Part B-Polymer Physics 1989, 27, 1029-1042.

(46) Fischer, E. Zusammenhänge zwischen der Kolloidstruktur kristalliner Hochpolymerer und ihrem Schmelz- und Rekristallisationsverhalten. Kolloid-Zeitschrift and Zeitschrift fur Polymere 1969, 231, 458-503.

(47) Peterlin, A. Thickening of polymer single crystals during annealing. Journal of Polymer Science Part B: Polymer Letters 1963, 1, 279-284, DOI: https://doi.org/10.1002/pol.1963.110010603.

(48) Mowry, S. W.; Rutledge, G. C. Atomistic Simulation of the $\alpha$ c-Relaxation in Crystalline Polyethylene. Macromolecules 2002, 35, 4539-4549, DOI: 10.1021/ma0118668.

(49) Lee, B.; Shin, T. J.; Lee, S. W.; Yoon, J.; Kim, J.; Youn, H. S.; Ree, M. Time-resolved Xray scattering and calorimetric studies on the crystallization behaviors of poly(ethylene terephthalate) (PET) and its copolymers containing isophthalate units. Polymer 2003, 44, 2509-2518, DOI: 10.1016/s0032-3861(03)00130-7.

(50) Hsiao, B.; Wang, Z.-g.; Yeh, F.; Gao, Y.; Sheth, K. Time-resolved X-ray studies of structure development in poly(butylene terephthalate) during isothermal crystallization. Polymer 1999, 40, 3515-3523, DOI: 10.1016/s0032-3861 (98)00573-4.

(51) Toda, A.; Taguchi, K.; Nozaki, K.; Guan, X.; Hu, W.; Furushima, Y.; Schick, C. Crystallization and melting of poly(butylene terephthalate) and poly(ethylene terephthalate) investigated by fast-scan chip calorimetry and small angle X-ray scattering. Polymer 2020, 192, 122303, DOI: 10.1016/j . polymer.2020.122303.

(52) Wang, Z.-G.; Wang, X.-H.; Hsiao, B. S.; Phillips, R. A.; Medellin-Rodriguez, F. J.; Srinivas, S.; Wang, H.; Han, C. C. Structure and morphology development in syndiotactic polypropylene during isothermal crystallization and subsequent melting. Journal of Polymer Science Part B: Polymer Physics 2001, 39, 2982-2995, DOI: 10.1002/polb. 10055.

(53) Gedde, U.; Mattozzi, A. Polyethylene morphology. Advances in Polymer Science 2004, 169, 29-73.

(54) Marand, H.; Huang, Z. Isothermal Lamellar Thickening in Linear Polyethylene: Correlation between the Evolution of the Degree of Crystallinity and the Melting Temperature. Macromolecules 2004, 37, 6492-6497, DOI: 10.1021/ma0497198.

(55) el Maaty, M.; Bassett, D. Evidence for isothermal lamellar thickening at and behind the growth front as polyethylene crystallizes from the melt. Polymer 2005, 46, 8682-8688.

(56) Kavesh, S.; Schultz, J. Lamellar and Interlamellar Structure in Melt-Crystallized Polyethylene .2. Lamellar Spacing, Interlamellar Thickness, Interlamellar Density, and Stacking Disorder. Journal of Polymer Science Part A-2-Polymer Physics 1971, 9, 85-114. 
(57) Schmidt-Rohr, K.; Spiess, H. Chain Diffusion Between Crystalline and Amorphous Regions in Polyethylene Detected by 2D Exchange C-13 Nmr. Macromolecules 1991, 24, 5288-5293.

(58) Hu, W. G.; Boeffel, C.; Schmidt-Rohr, K. Chain Flips in Polyethylene Crystallites and Fibers Characterized by Dipolar13C NMR Volume 32, Number 00, p 000. Macromolecules 1999, 32, 1714-1714, DOI: 10.1021/ma982401t.

(59) Bärenwald, R.; Goerlitz, S.; Godehardt, R.; Osichow, A.; Tong, Q.; Krumova, M.; Mecking, S.; Saalwächter, K. Local Flips and Chain Motion in Polyethylene Crystallites: A Comparison of Melt-Crystallized Samples, Reactor Powders, and Nanocrystals. Macromolecules 2014, 47, 5163-5173, DOI: 10.1021/ma500691k.

(60) Bärenwald, R.; Goerlitz, S.; Godehardt, R.; Osichow, A.; Tong, Q.; Krumova, M.; Mecking, S.; Saalwächter, K. Correction to Local Flips and Chain Motion in Polyethylene Crystallites: A Comparison of Melt-Crystallized Samples, Reactor Powders, and Nanocrystals (vol 47, pg 5163, 2014). Macromolecules 2014, 47, 7677-7678, DOI: $10.1021 / \mathrm{ma} 5020963$.

(61) de Langen, M.; Prins, K. O. Mobility of polyethylene chains in the orthorhombic and hexagonal phases investigated by NMR. Chemical Physics Letters 1999, 299, 195-200, DOI: https ://doi .org/10.1016/S0009-2614(98)01250-0.

(62) Auriemma, F.; De Rosa, C.; Corradini, P. Solid mesophases in semicrystalline polymers: Structural analysis by diffraction techniques; Advances in polymer science; 2005; Vol. 181; pp 1-74.

(63) Luo, C.; Sommer, J. Role of Thermal History and Entanglement Related Thickness Selection in Polymer Crystallization. ACS Macro Letters 2016, 5, 30-34, DOI: 10.1021/acsmacrolett.5b00668.

(64) Iwata, K. Role of entanglement in crystalline polymers 1. Basic theory. Polymer 2002, 43, 6609-6626, DOI: 10.1016/S0032-3861(02) 00524-4.

(65) Mark, J. E. Physical Properties of Polymers Handbook; Springer: New York, 2007; DOI: $10.1007 / 978-0-387-69002-5$.

(66) Save, M.; Schappacher, M.; Soum, A. Controlled Ring-Opening Polymerization of Lactones and Lactides Initiated by Lanthanum Isopropoxide, 1. General Aspects and Kinetics. Macromol. Chem. Phys 2002, 203, 889-899, DOI: 10.1002/1521-3935(20020401) 203:5/6<889: :AID-MACP889>3 . 0 . C0; 2-0.

(67) Kern, W.; Baader, H.; Cherdron, H.; Hohr, L.; Deibig, H.; Giefer, A.; Jaacks, V.; Wildenau, A. Polyoxymethylene. Angewandte Chemie-International Edition 1961, 73, $177-224$.

(68) Stohler, F.; Berger, K. Stabilization of Polyacetals. Angewandte Makromolekulare Chemie 1990, 176, 323-332, DOI: 10.1002/apmc.1990.051760125. 
(69) Polyoxymethylene (POM) Heat Capacity, Enthalpy, Entropy, Gibbs Energy: Datasheet from "The Advanced THermal Analysis System (ATHAS) Databank - Polymer Thermodynamics" Release 2014 in SpringerMaterials. https ://materials . springer.com/ polymerthermodynamics/docs/athas_0069, Copyright 2014 Springer-Verlag Berlin Heidelberg \& Marek Pyda, Part of SpringerMaterials accessed 2020-07-21.

(70) Poly(epsilon-caprolactone) (PCL) Heat Capacity, Enthalpy, Entropy, Gibbs Energy: Datasheet from "The Advanced THermal Analysis System (ATHAS) Databank Polymer Thermodynamics" Release 2014 in SpringerMaterials. https://materials . springer.com/polymerthermodynamics/docs/athas_0049, Copyright 2014 SpringerVerlag Berlin Heidelberg \& Marek Pyda, Part of SpringerMaterials accessed 2020-07-21.

(71) Polyoxyethylene (POE) Heat Capacity, Enthalpy, Entropy, Gibbs Energy: Datasheet from "The Advanced THermal Analysis System (ATHAS) Databank - Polymer Thermodynamics" Release 2014 in SpringerMaterials. https ://materials . springer.com/ polymerthermodynamics/docs/athas_0068, Copyright 2014 Springer-Verlag Berlin Heidelberg \& Marek Pyda, Part of SpringerMaterials accessed 2020-07-21.

(72) Albrecht, T.; Strobl, G. Temperature-Dependent Crystalline-Amorphous Structures in Linear Polyethylene - Surface Melting and the Thickness of the Amorphous Layers. Macromolecules 1995, 28, 5827-5833. 


\section{Acknowledgement}

We thank Katrin Herfurt for technical help with calorimetric measurements and Anika Wurl for performing the rheological measurements. Funding was provided by Deutsche Forschungsgemeinschaft (DFG, German Research Foundation)-Projektnummer 189853844-TRR 102, project A1.

\section{Author contributions}

M. Schulz performed and designed experiments (SAXS and optical microscope), analysed and visualized data. M. Schäfer performed and analysed the NMR-experiments. T. ThurnAlbrecht and K. Saalwächter conceived and supervised the project. M. Schulz and T. Thurn-Albrecht wrote the manuscript. All authors discussed results and commented on the manuscript.

\section{Competing interests}

The authors declare no competing interests

\section{Correspondence}

Correspondence and requests for materials should be addressed to K. S. (kay.saalwaechter@physik.unihalle.de) and T. T.-A. (thomas.thurn-albrecht@physik.uni-halle.de)

\section{Supplementary Information}

- Characterization of ICD in poly(oxymethylene) by NMR

- Complementary rheological measurements

- Calculation of the timescales $\tau_{l c},\left\langle\tau_{c}\right\rangle$ and $\tau_{\text {stem }}$

- Structural analysis by SAXS - method and exemplary data

- SAXS data shown in Fig. 3 and Fig. 4a in digital format. 


\section{Supplementary Files}

This is a list of supplementary files associated with this preprint. Click to download.

- SAXSdataFig34a.xlsx

- SI15.pdf 